\title{
Constraints on the maximum mass of quark star and the GW 190814 event
}

\author{
Takol Tangphati ${ }^{1, \mathrm{a}} \mathbb{D}$, Indrani Karar $^{2, \mathrm{~b}}$, Anirudh $\operatorname{Pradhan}^{3, \mathrm{c}}{ }_{\mathbb{C}}$, Ayan Banerjee $^{4, \mathrm{~d}}(\mathbb{C}$ \\ ${ }^{1}$ Theoretical and Computational Physics Group, Theoretical and Computational Science Center (TaCS), Faculty of Science, \\ King Mongkut's University of Technology Thonburi, Prachauthid Rd., Bangkok 10140, Thailand \\ ${ }^{2}$ Department of Registrar, Kalyani University, Nadia, West Bengal, India \\ ${ }^{3}$ Centre for Cosmology, Astrophysics and Space Science, GLA University, Mathura, Uttar Pradesh 281 406, India \\ ${ }^{4}$ Astrophysics and Cosmology Research Unit, School of Mathematics, Statistics and Computer Science, University of KwaZulu-Natal, \\ Private Bag X54001, Durban 4000, South Africa
}

Received: 17 November 2021 / Accepted: 11 January 2022 / Published online: 21 January 2022

(C) The Author(s) 2022

\begin{abstract}
Gravitational-waves (GWs) data have widely been used for testing preferred modified gravity theories. In this paper, we investigate the possibility of testing them in the strong gravity regime by looking at the properties of compact objects in dense matter physics. In this direction modified gravity theories such as $f(R, T)$ gravity can be tested with the recently discovered compact binary merger, GW 190814 , containing a compact object with mass $2.50-2.67 M_{\odot}$. By considering these constraints on maximum mass of such an object, we predict the existence of quark stars (QSs) made of quark matter in the color-flavor-locked (CFL) phase of color superconductivity. Such a state is significantly more bound than ordinary quark matter and enhances the possibility of the existence of a pure stable QS. We focus on the following aspects in particular: mass-radius profile, mass-central mass density relation, compactness and the corresponding effective adiabatic index for stability related issues. Our result implies that predicted properties for QSs are well consistent with GW 190814 observational data that helps us to impose constraints on the theoretical models of dense nuclear matter.
\end{abstract}

\section{Introduction}

For over a century, classical general relativity (CGR) has rewarded us richly in the areas of astrophysics and cosmology. As we enter a golden age of observations, with higher precision and advanced technologies, there is a greater demand on CGR. Einstein's formulation of gravitation has

\footnotetext{
a e-mail: takoltang@ gmail.com

be-mail: indrani.karar08@gmail.com

c e-mail: pradhan.anirudh@gmail.com

de-mail: ayanbanerjeemath@gmail.com (corresponding author)
}

continued to bear out these observations, the most notable being the detection of gravitational waves [1] and the photographing of the shadow of a black hole [2]. Despite the many successes of CGR, several shortcomings have arisen in relativistic astrophysics, dust and gas reservoirs some of which include the end-states of continued gravitational collapse, high-redshift star-forming galaxies and equation of state of ultra-high densities. On the cosmological front, CGR has been burdened with the flatness problem, the horizon problem, the observed acceleration of the universe and the origin of the Big Bang.

In order to overcome these pathologies, there were various attempts to modify CGR. This gave rise to a spectrum of modified theories of gravity, including $f(R), f(R, T)$, Lovelock gravity, Einstein-Gauss-Bonnet (EGB) gravity, amongst others. Modifications to CGR not only attempted to plug the holes in Einstein's formulation of gravity but have extended the space of exact and numerical solutions. For example, $f(R)$ gravity was proposed by Starobinsky to account for the observed late-time acceleration of the Universe without requiring exotic matter such as dark energy or scalar fields [3]. The $f(R)$ theory of gravitation requires the incorporation into the action of a polynomial in the Ricci scalar. The leading contender of the most natural generalisation of CGR in the strong-field domain within the $f(R)$ framework is the so-called $R$-squared theory in which $f(R)=R+a R^{2}$. A comparative study of neutron and strange stars oscillations in CGR and $R^{2}$ gravity was carried out by Staykov et al. [4]. In this work they demonstrated that In this paper, we investigated neutron and strange stars oscillations in GR and $R^{2}$ gravity. By employing the Cowling approximation over a wide spectrum of EoS's with varying stiffnesses, they showed the observed maximum deviation 
between the $f$-mode frequencies in CGR models compared to their $R^{2}$ counterparts is up to $10 \%$ is sensitive to the $R^{2}$ parameter $a$. In a separate study, Staykov et al. [5] studied slowly rotating neutron and strange stars in $R^{2}$ gravity by employing two different hadronic equations of state and a strange matter equation of state. Numerical solutions of the governing equations revealed that the $R^{2}$ neutron star moment of inertia can be up to $30 \%$ larger than its CGR counterpart. This could serve as a possible signature for distinguishing CGR and $f(R)$ theories via observations. The serious drawback of $f(R)$ theory is the appearance of higher derivative terms which correspond to ghosts.

In this work we attempt to constrain the maximum mass of a quark star in $f(R, T)$ gravity by using data obtained from the GW 190814 event. Stellar modeling within the framework of $f(R, T)$ gravity has become a popular choice amongst relativists which was proposed by Harko et al. [6]. The action in this formalism is a function of the Ricci scalar $R$ and the trace of the energy momentum tensor $T$ and has been christened $f(R, T)$ gravity in the literature. While the the equations of motion are indeed second order, the main drawback is the violation of the conservation of energy. This seemingly violation of the law of energy conservation can be attributed to the geometry of spacetime, in particular the curvature of spacetime as compared to its Newtonian counterpart. In the recent past, there have been many inroads made in establishing compact objects in $f(R, T)$ gravity including the Mazur-Mottola gravastar model as a viable alternative to black holes [7], modeling of isothermal fluids [8], studying the stability of neutron stars by adopting a polytropic equation of state (EoS) [9], modeling charged stellar objects [10], to name a few.

It is well-known that the study of compact objects such as neutron stars, pulsars and quark stars in both CGR and modified theories of gravity have leap-frogged from mathematical excursion of the governing equations into mainstream observations of these astrophysical bodies. One observational event in particular has served as a bedrock for testing such models and is useful to compare possible signatures of modified gravity to their classical counterparts. The so-called gravitational wave events recorded by the LIGOVirgo collaboration [11] has been drawn into the context of Einstein-dilaton-Gauss-Bonnet (EdGB) and dynamical Chern-Simons gravity. The signals detected by the binary black hole narrowed down the bounds on the EdGB coupling constant to $\alpha_{E d G B}^{1 / 2} \leq 5.6 \mathrm{~km}$ [12]. On the cosmological front, the compatibility of Einstein-Gauss-Bonnet gravity with GW170817 event has led to a reformulation of EGB inflation [13]. The gravitational waves generated in the coalescing of two neutron stars and the observation that their arrival time coincided with gamma rays required that gravitational wave speed be $c_{T}^{2} \approx 1$ in natural units. This in turn constrained the functional forms for the scalar Gauss-Bonnet coupling function $\zeta(\phi)$ and the scalar potential $V(\phi)$.

The discovery of a compact object with a mass greater than $\sim 2 M_{\odot}$ via the GW 190814 binary coalescence has prompted researchers to mine extended theories of gravity to account for stellar characteristics such as mass-radius relations of such a body. It has been shown that in the presence of rotation, the masses of neutron stars can exceed $\sim 2.6 M_{\odot}$ for particular EoS's and still be compatible with observations from the GW 190814 event [14].

The paper is organized as follows: in Sect. 2 we briefly reviewed the field equations for $f(R, T)$ gravity. For spherically symmetric solutions of these equations, the modified Tolman-Oppenheimer-Volkoff (TOV) equations are derived in Sect. 3. In Sect. 4, we present the EoS for CFL phase which is the true ground state of hadronic matter at asymptotically large densities. The interior solution is being matched with the exterior Schwarzschild vacuum solution with appropriate boundary conditions. In Sect. 5, we solve the modified TOV equations numerically and determine the mass-radius relations for QSs for two different sets of parameters. We also investigate the stability of QSs in the same section. We draw final conclusions from our results in in Sect. 6. Here we adopt the signature $(+,-,-,-)$, and utilise geometric units by setting the gravitational constant $G$ and the speed of light $c$ to unity, that is $G=c=1$.

\section{Field equations and set up}

Following Harko et al. [6], the action of the $f(R, T)$ modified gravity in $4 D$ spacetime is given by

$S=\int d^{4} x \sqrt{-g}\left[\frac{1}{16 \pi} f(R, T)+\mathcal{L}_{m}\right]$,

where $f(R, T)$ is an arbitrary function of the Ricci scalar $R$ and of the trace of the stress-energy tensor $T$, respectively. From the matter Lagrangian density $\mathcal{L}_{m}$, we defined the energy-momentum tensor of the matter as

$T_{\mu \nu}=-\frac{2}{\sqrt{-g}} \frac{\delta\left(\sqrt{-g} \mathcal{L}_{m}\right)}{\delta g^{\mu \nu}}=g_{\mu \nu} \mathcal{L}_{m}-2 \frac{\partial \mathcal{L}_{m}}{\partial g^{\mu \nu}}$.

There is an implicit assumption that $\mathcal{L}_{m}$ depends only on the metric components $g_{\mu \nu}$ and not on its derivatives. Now, varying the action (1) with respect to the metric tensor, the field equations are obtained as

$$
\begin{aligned}
& f_{R}(R, T) R_{\mu \nu}-\frac{1}{2} f(R, T) g_{\mu \nu}+\left(g_{\mu \nu} \square-\nabla_{\mu} \nabla_{\nu}\right) \\
& \quad \times f_{R}(R, T)=8 \pi T_{\mu \nu}-f_{T(R, T)} T_{\mu \nu}-f_{T(R, T)} \Theta_{\mu \nu},
\end{aligned}
$$

where $f_{R}(R, T)=\partial f(R, T) / \partial R, f_{T}(R, T)=\partial f(R, T) / \partial T$, $\square \equiv \partial_{\mu}\left(\sqrt{-g} g^{\mu \nu} \partial_{\nu}\right) / \sqrt{-g}, R_{\mu \nu}$ is the Ricci tensor, $\nabla_{\mu}$ 
denotes the covariant derivative with respect to the metric $g_{\mu \nu}$ and $\Theta_{\mu \nu}=g^{\alpha \beta} \delta T_{\alpha \beta} / \delta g^{\mu \nu}$. The covariant derivative of the field Eq. (3) gives

$$
\begin{gathered}
\nabla^{\mu} T_{\mu \nu}=\cdot \frac{f_{T}(R, T)}{8 \pi-f_{T}(R, T)}\left[\left(T_{\mu \nu}+\Theta_{\mu \nu}\right) \nabla^{\mu}\right. \\
\left.\times \ln f_{T}(R, T) \nabla^{\mu} \Theta_{\mu \nu}-(1 / 2) g_{\mu \nu} \nabla^{\mu} T\right]
\end{gathered}
$$

which is significant in discussing energy properties of the model.

Assuming that the matter content is a perfect fluid, the stress tensor is given by

$T_{\mu \nu}=(\rho+P) u_{\mu} u_{\nu}-P g_{\mu \nu}$,

where $u^{\mu}$ is the 4-velocity which satisfies the condition $u^{\mu} u_{\mu}=1$. With this $\rho$ and $P$ are the matter energy-density and the isotropic pressure of the fluid, respectively. For the perfect fluid we can fix the matter Lagrangian as $\mathcal{L}_{m}=-P$ (see [6] for details), which gives

$\Theta_{\mu \nu}=-2 T_{\mu \nu}-P g_{\mu \nu}$.

To proceed further, we assume the simplest linear functional form of $f(R, T)$ model i.e., an ansatz $f(R, T)=$ $R+2 \lambda T$ [6]. Such assumption has been considered in many cosmological findings, see Refs. [15-17] for more. With this assumption, the field Eq. (3) turns out to be

$G_{\mu \nu}=8 \pi T_{\mu \nu}+\lambda T g_{\mu \nu}+2 \lambda\left(T_{\mu \nu}+P g_{\mu \nu}\right)$,

where $G_{\mu \nu}$ is the Einstein tensor and $\lambda$ is an arbitrary constant. Moreover, the Einstein gravity is recovered when $\lambda=0$. Since, Eq. (4) leads to the form

$(8 \pi+2 \lambda) \nabla^{\mu} T_{\mu \nu}=-2 \lambda\left[\nabla^{\mu}\left(p g_{\mu \nu}\right)+\frac{1}{2} g_{\mu \nu} \nabla^{\mu} T\right]$.

Since the $f(R, T)$ gravity is a curvature-matter coupling theory and leads to a non-conservation of the energymomentum tensor. The role of curvature-matter coupling as an exchange of energy and momentum between both, and leads to the appearance of an extra acceleration [18].

\section{Structural equation of star}

For the spacetime metric we consider here static and spherically symmetric line element $\left(d s^{2}=g_{\nu \mu} d x^{\nu} d x^{\mu}\right.$, where $\nu, \mu=0,1,2,3)$,

$d s^{2}=e^{\Phi(r)} d t^{2}-e^{\Lambda(r)} d r^{2}-r^{2}\left(d \theta^{2}+\sin ^{2} \theta d \phi^{2}\right)$,

where $\Phi(r)$ and $\Lambda(r)$ are arbitrary functions of the radial coordinate, $r$. Considering the energy-momentum tensor of the perfect fluid (5) and using the Eqs. (9) and (7), the (tt) and $(r r)$ components for $f(R, T)$ gravity reads [19]:

$e^{-\Lambda}\left(\frac{\Lambda^{\prime}}{r}-\frac{1}{r^{2}}\right)+\frac{1}{r^{2}}=(8 \pi+3 \lambda) \rho-\lambda P$,

$e^{-\Lambda}\left(\frac{\Phi^{\prime}}{r}+\frac{1}{r^{2}}\right)-\frac{1}{r^{2}}=(8 \pi+3 \lambda) P-\lambda \rho$,

and the hydrostatic equilibrium equation for $f(R, T)$ gravity reads [19]

$P^{\prime}+(\rho+P) \frac{\Phi^{\prime}}{2}=\frac{\lambda}{8 \pi+2 \lambda}\left(P^{\prime}-\rho^{\prime}\right)$,

where prime denotes the derivative with respect to $r$. Here, we write down the Eqs. (10) and (12) in a more conventional form by introducing the transformation $e^{-2 \Lambda}=1-\frac{2 m(r)}{r}$ such that $m(r)$ is identified with the active gravitational mass of the star and consequently generates the forms

$m^{\prime}(r)=4 \pi r^{2} \rho+\frac{\lambda(3 \rho-p) r^{2}}{2}$,
$P^{\prime}(r)=-\frac{(\rho+P)\left[4 \pi p r+\frac{m}{r^{2}}-\frac{\lambda(\rho-3 P) r}{2}\right]}{\left(1-\frac{2 m}{r}\right)\left[1-\frac{\lambda}{8 \pi+2 \lambda}\left(1-\frac{d \rho}{d P}\right)\right]}$,

which will be useful for our numerical computation. These equations are nothing else than ordinary TOV equations when $\lambda=0$. Further, by choosing an EoS, we compute massradius relation for different sets of parameters. Here, we consider QSs formed by quark matter in the color-flavor-locked (CFL) phase of color superconductivity.

\section{Equations of state for quark matter and boundary conditions}

\subsection{Color flavor locked quark matter}

Quantum chromodynamics (QCD) is the theory of quarks and gluons that describes the strong interactions and predict that quark matter at sufficiently high baryon density and low temperature becomes a color superconductor (CSC) [20-22]. Such a state depends sensitively on the number of quark flavors and their masses [23,24]. Among some major developments on quark pairings in the past decade it is believed that at asymptotically high densities, QCD favors a maximally symmetric phase of superconducting quark matter called the CFL phase [25]. In nature, such conditions may be realized in the cores of cold compact stars, see Ref. [26].

In this situation, it is useful to consider an EoS of a strange star described by a CFL model satisfying several observational constraints from various known pulsars, and massradius estimates derived from different data sources. It is generally agreed that the CFL phase is the real ground state of QCD at asymptotically large densities, and even if the quark masses are unequal $[27,28]$. The EoS for the CFL phase is 
charge neutral which can be obtained in the framework of the MIT bag model. The thermodynamic potential for electric and color charge neutral CFL quark matter to order $\Delta^{2}$ is given by [29]

$$
\begin{aligned}
\Omega_{C F L}= & -\frac{3 \Delta^{2} \mu^{2}}{\pi^{2}}+\frac{6}{\pi^{2}} \int_{0}^{\gamma_{F}} p^{2}(p-\mu) d p \\
& +\frac{3}{\pi^{2}} \int_{0}^{\gamma_{F}} p^{2}\left(\sqrt{p^{2}+m_{s}^{2}}-\mu\right) d p+B,
\end{aligned}
$$

where $B$ is the MIT bag constant with $\Delta$ denotes the color superconducting gap and $\mu$ is the quark chemical potential. The first term is the contribution of the CFL condensate to $\Omega_{C F L}$, while the next two terms coming from the fictitious non-paired state proportional to the volume of the Fermi sphere in which all quarks have a common Fermi momentum $\gamma_{F}$, with $\gamma_{F}$ chosen to minimize the thermodynamic potential of the fictional unpaired quark matter [30].

Since, the term $\gamma_{F}$ is given by

$$
\gamma_{F}=\left[\left(2 \mu-\sqrt{\mu^{2}+\frac{m_{s}^{2}-m_{u}^{2}}{3}}\right)^{2}-m_{u}^{2}\right]^{1 / 2},
$$

where $\mu=\left(\mu_{s}+\mu_{u}+\mu_{d}\right) / 3$ is the average quark chemical potential, and $m_{s}$ and $m_{u}$ are strange and up quark masses, respectively. For massless up and down quarks we have

$\gamma_{F}=2 \mu-\sqrt{\mu^{2}+\frac{m_{s}^{2}}{3}} \sim \mu-\frac{m_{s}^{2}}{6 \mu}$.

Studying the pairing ansatz in the CFL phase [31]

$n_{u}=n_{r}, \quad n_{d}=n_{g}, \quad$ and $\quad n_{s}=n_{b}$

where $n_{u}, n_{d}, n_{s}$ and $n_{r}, n_{g}, n_{b}$ are flavor and color number densities respectively. These quantities can be obtained for an isotropic system from the thermodynamic potential through the relations

$P=-\Omega_{C F L}$ and $n_{u}=n_{d}=n_{s}=\frac{\gamma_{F}^{3}+2 \Delta^{2} \mu}{\pi^{2}}$,

and the energy density $\epsilon$ can be easily derived at zero which reads [32]

$\epsilon=3 \mu n_{B}-P$,

where $n_{B}$ is the baryon number density. Interestingly, when $m_{s} \rightarrow 0$ the particle densities become equal, and we recover the well known form of MIT bag model $\epsilon=3 P+4 B$. The extra term dependent on $\Delta^{2}$ in Eq. (15); picks up an additional term from CFL contribution as $\epsilon=3 P+4 B-6 \Delta^{2} \mu^{2} / \pi^{2}$. Interestingly, this situation turns out to be more complicated when $m_{s} \neq 0$, because the equation of state must be calculated numerically. To order $\Delta^{2}$ and $m_{s}^{2}$ the pressure and energy density can be written as [32]

$$
\begin{gathered}
P=\frac{3 \mu^{4}}{4 \pi^{2}}+\frac{9 \beta \mu^{2}}{2 \pi^{2}}-B, \\
\epsilon=\frac{9 \mu^{4}}{4 \pi^{2}}+\frac{9 \beta \mu^{2}}{2 \pi^{2}}+B,
\end{gathered}
$$

where

$\beta=-\frac{m_{s}^{2}}{6}+\frac{2 \Delta^{2}}{3}$.

With the use of above expression the energy density $\epsilon$ of the quark matter in the CFL phase can be obtained as

$\epsilon=3 P+4 B-\frac{9 \beta}{\pi^{2}}\left\{\left[\frac{4 \pi^{2}(B+P)}{3}+9 \beta^{2}\right]^{1 / 2}-3 \beta\right\}$.

We see from Eq. (24) that there are three free parameters in the EoS $m_{s}, \Delta$ and $B$, respectively. The values of all the free parameters are considered in this paper fall inside the stability windows, see [33].

\subsection{Boundary conditions}

Solving the TOV equations (13) and (14) for a compact star, we perform numerical integration and maintain the regularity inside the fluid sphere with appropriate boundary conditions:

$m(0)=0, \quad \rho(0)=\rho_{c}, \quad P(0)=P_{c}$.

where $r=R$ is identified as the surface of the star with $P_{c}$ is the central pressure and $\rho_{c}$ is the central energy density, respectively. At this point the interior solution is matched at the boundary $r=R$ to the asymptotically flat vacuum exterior Schwarzschild solution which are connected by $e^{\Phi(R)}=e^{-\Lambda(R)}=1-2 M / R$, where $M=m(r=R)$ representing the total mass of the QS.

\section{Numerical results}

Considering the EoS (see Sect. 4), we numerically solve the TOV equations (13) and (14) for a given set of central energy densities. To get an idea of how quark stars might be, we take the values of model parameters on a reasonable range and obtain the so-called mass-radius $(M-R)$ and masscentral energy density $\left(M-\rho_{c}\right)$ relationships for the given EoS. The mass of the star is measured in units of solar masses $M_{\odot}$, the radius of the stars in $\mathrm{Km}$, while both the pressure and energy density profiles are measured in $\mathrm{MeV} / \mathrm{fm}^{3}$. Through this process, we discuss two sets of solutions characterizing the model (i) variation of coupling parameter $\delta$ and (ii) variation of bag constant $B=(57-92) \mathrm{MeV} / \mathrm{fm}^{3}$ [34] to see the effect of modified gravity on $(M-R)$ relations. The other 

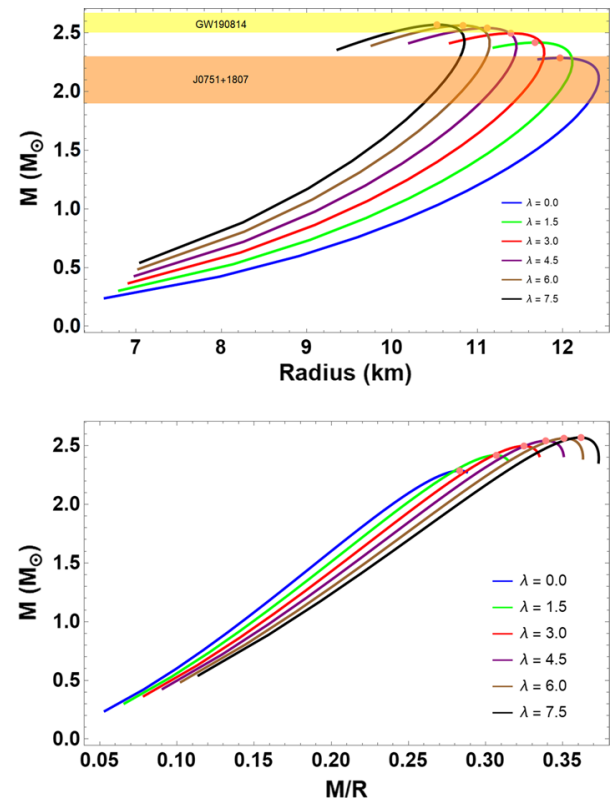

Fig. 1 Mass-radius $(M-R)$ relation and compactness $M-M / R$ for QSs have been plotted in the $f(R, T)=R+2 \lambda T$ gravity compared with GR considering the CFL EoS. For plotting the figures we have chosen $B=57 \mathrm{MeV} / \mathrm{fm}^{3}, \lambda=1.0, m_{s}=100 \mathrm{MeV}$ and different values of $\lambda=\{0.0,1.5,3.0,4.5,6.0,7.5\}$, respectively. The horizontal bars correspond to the constraints imposed by the secondary component of the GW 190814 event (yellow) [35] and PSR J0751+1807 (orange) [36]. Each color represents a different $M-R$ relation with parameters mentioned in Table 1
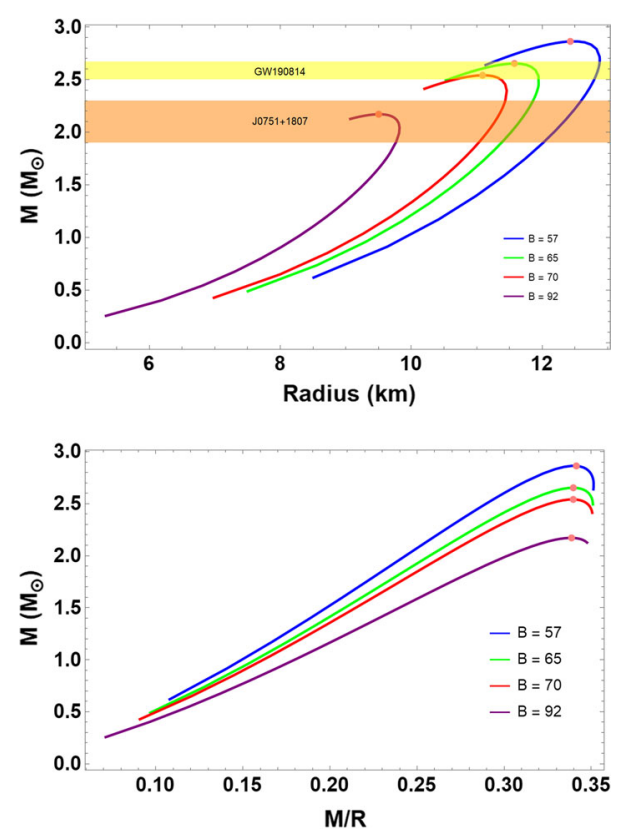

Fig. 2 Mass-radius $(M-R)$ relation and compactness $M-M / R$ of QSs have been plotted for $\lambda=4.5 \mathrm{MeV}, m_{s}=50 \mathrm{MeV}, \Delta=120 \mathrm{MeV}$ and different values of $B=\{57,65,70,92\} \mathrm{MeV} / \mathrm{fm}^{3}$. The horizontal bars correspond to the constraints imposed by the GW 190814 event (yellow) [35] and PSR J0751+1807 (orange) [36]. Each color represents a different $M-R$ relation with parameters mentioned in Table 2
Table 1 Properties of quark stars in $f(R, T)$ gravity for different values of $\lambda$. Estimated values of maximum masses and their corresponding radii have been tabulated for specific central mass density $\rho_{c}$. The compactness $M / R$ is measured as a dimensionless quantity. Fitting parameters for CFL EoS can reveal some features of the influence of $f(R, T)$ gravity on the QSs configurations, whereas $\lambda=0$ leading to the GR solution

\begin{tabular}{lllll}
\hline$\lambda$ & $\begin{array}{l}M \\
M_{\odot}\end{array}$ & $\begin{array}{l}R \\
\mathrm{~km}\end{array}$ & $\begin{array}{l}\rho_{c} \\
\mathrm{MeV} / \mathrm{fm}^{3}\end{array}$ & $M / R$ \\
\hline 0.0 & 2.29 & 11.97 & 930 & 0.283 \\
1.5 & 2.42 & 11.68 & 804 & 0.307 \\
3.0 & 2.50 & 11.39 & 704 & 0.325 \\
4.5 & 2.54 & 11.12 & 630 & 0.339 \\
6.0 & 2.56 & 10.83 & 581 & 0.351 \\
7.5 & 2.57 & 10.53 & 556 & 0.362 \\
\hline
\end{tabular}

parameters $m_{s}$ and $\Delta$ remain fixed and measured in units of $\mathrm{MeV}$.

\subsection{Mass-radius relation}

We have studied the dependence of the maximum mass of QSs on the value of $\lambda$, as shown in Fig. 1. Each curves have been plotted for $B=70 \mathrm{MeV} / \mathrm{fm}^{3}, m_{s}=50 \mathrm{MeV}$ and $\Delta=120 \mathrm{MeV}$ and different values of coupling constant $\lambda=$ $\{0.0,1.5,3.0,4.5,6.0,7.5\}$. From the upper panel, it can be found that the maximum masses of QSs are around $2.42-$ $2.57 M_{\odot}$ and the corresponding radii are $10.53-11.68 \mathrm{~km}$. Moreover, the largest $M_{\max }$ values can explain the existence of the secondary component of the GW 190814 event, which has mass $2.50-2.67 M_{\odot}$ [35]. We find that for CFL EoS supporting $M_{\max }>2.5$ with radii in the range $10.53-11.39$ for the considered parameter sets (see Table 1), which is consistent with GW 190814 data. In this case the maximum mass for GR $(\lambda=0)$ can't exceed $2.5 M_{\odot}$ limit but can explain the existence of PSR J0751+1807 with $2.1 \pm 0.2 M_{\odot}$ (orange) [36], see Fig. 1. These results show that the influence of $\lambda$ on the maximum mass, the minimum radius and the maximum compactness is achieved, see Fig. 1 and Table 1 for more details. The compactness of the star, $M / R$, at the maximum mass/minimum radius is shown in the lower panel of Fig. 1.

In Fig. 2, we follow the same procedure presented in Fig. 1 to obtain the mass-radius $(M-R)$ relations and the compactness of QSs within the framework of the $f(R, T)$ gravity model. The choice of the parameters are $\lambda=4.5 \mathrm{MeV}$, $m_{s}=50 \mathrm{MeV}, \Delta=120 \mathrm{MeV}$ and different values of bag constant in the region $57 \leq B \leq 92 \mathrm{MeV} / \mathrm{fm}^{3}$. By varying $B$ it is evident that the maximum mass and radius increase their values up to $2.86 M_{\odot}$ with radius $R=12.43 \mathrm{Km}$. Moreover, the maximum masses calculated for $B=65,70 \mathrm{MeV} / \mathrm{fm}^{3}$ sets are about 2.65-2.54 $M_{\odot}$ with $11.09-11.57 \mathrm{Km}$, which 
Table 2 Properties of quark stars in $f(R, T)$ gravity for different values of bag constant $B$. All parameters for calculations are chosen according to the discussion given in Fig. 2

\begin{tabular}{lllll}
\hline$B$ & $\begin{array}{l}M \\
M_{\odot}\end{array}$ & $\begin{array}{l}R \\
\mathrm{~km}\end{array}$ & $\begin{array}{l}\rho_{c} \\
\mathrm{MeV} / \mathrm{fm}^{3}\end{array}$ & $M / R$ \\
\hline 57 & 2.86 & 12.43 & 521 & 0.341 \\
65 & 2.65 & 11.57 & 586 & 0.340 \\
70 & 2.54 & 11.09 & 642 & 0.340 \\
92 & 2.17 & 9.50 & 873 & 0.339 \\
\hline
\end{tabular}

are in accord with the observed mass of the secondary compact object in GW 190814 (Yellow), see Table 2. In addition to the constraints from PSR J0751+1807 (orange), it is evident from the Fig. 2 that the model is satisfied for $B=92$ $\mathrm{MeV} / \mathrm{fm}^{3}$ i.e., highest value of bag constant. It should be noted that corresponding to the most interacting quark matter leads to a less compact QS modeled here, see Table 2.

\subsection{The stability criterion and the adiabatic indices}

We now consider the onset of instability is the $M\left(\rho_{c}\right)$ method, where the stellar mass $M$ against the central energy density $\epsilon_{c}$ is varied for different values of coupling constant $\lambda$ and bag constant $B$. Following the definition of static stability criterion [37,38], we can write

$$
\begin{aligned}
& \frac{d M}{d \rho_{c}}<0 \rightarrow \text { unstable configuration } \\
& \frac{d M}{d \rho_{c}}>0 \rightarrow \text { stable configuration, }
\end{aligned}
$$

to be satisfied by all stellar configurations. Note that this is a necessary condition but not sufficient where $\left(M_{\max }, R_{M_{\max }}\right)$ is a boundary separating the stable configuration region from the unstable one indicated by the inequalities (26), (27), respectively. The Fig. 3 indicates the mass versus central density curve which grows with the increasing values of mass in agreement with the $M-R$ profiles given in Figs. 1 and 2 , respectively. It is seen from the figure that the stability of QSs is guaranteed in the region where $d M / d \rho_{c}>0$ and the circle on each $M-\rho_{c}$ curve describing the maximum-mass stellar configuration.

On the other hand, we study the adiabatic index $(\gamma)$ which is related with the stability of stars. Following Chandrasekhar $[39,40]$ in his two seminal works, the dynamical stability of the star has been analyzed based on the variational method. The adiabatic index, which appears in the stability formula, reads

$\gamma \equiv\left(1+\frac{\rho}{P}\right)\left(\frac{d P}{d \rho}\right)_{S}$,

where $d P / d \rho$ is the speed of sound. The subscript $S$ in (28) indicates at constant specific entropy. In general, Eq. (28) is a
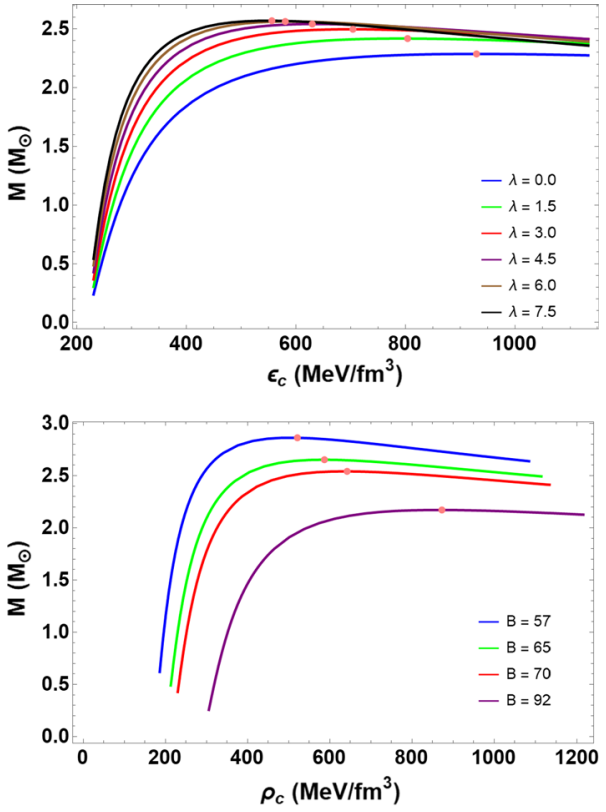

Fig. $3 M-\rho_{c}$ relationship is presented for the selected EoS together with the same set of parameters in Figs. 1 and 2, respectively

dimensionless quantity which measuring the stiffness of the EoS for a spherical relativistic fluid.

For relativistic polytropes the value of $\gamma$ should be $\gamma>$ $\gamma_{c r}=4 / 3$ depending on the ratio $\rho / P$ at the centre of the star, where $\gamma_{c r}$ is the critical adiabatic index [41]. For the selected EoS, we see from Fig. 4 that $\gamma>4 / 3 \sim 1.33$ for the given sets of parameters. This means that the solution branch we consider in this work is stable against the radial adiabatic infinitesimal perturbations.

\section{Concluding remarks}

In this work we have analyzed QSs that consists of quark matter in the color-flavor-locked (CFL) phase of color superconductivity. It is generally argued that CFL is likely to be the ground state of matter, at sufficiently high densities and low temperatures. In spite of this we have shown the effects of quark matter on the QS mass-radius relation by using a modified TOV equation derived from the $f(R, T)$ gravity theory. If we consider the limit $\lambda \rightarrow 0$, one can recover the standard hydrostatic equilibrium theory coming from GR.

The key point of the method is to explore the compatibility of QSs in the CFL phase with a set of observational constraints obtained from astronomical data, specially focusing on the recent discovery of a compact binary merger, GW 190814, containing a compact object with mass 2.50-2.67 $M_{\odot}$. Aiming this we vary two sets of parameters (a) mattergeometry coupling constant $\lambda$ and (b) the bag constant $B$, separately. It turns out that by increasing the positive value 

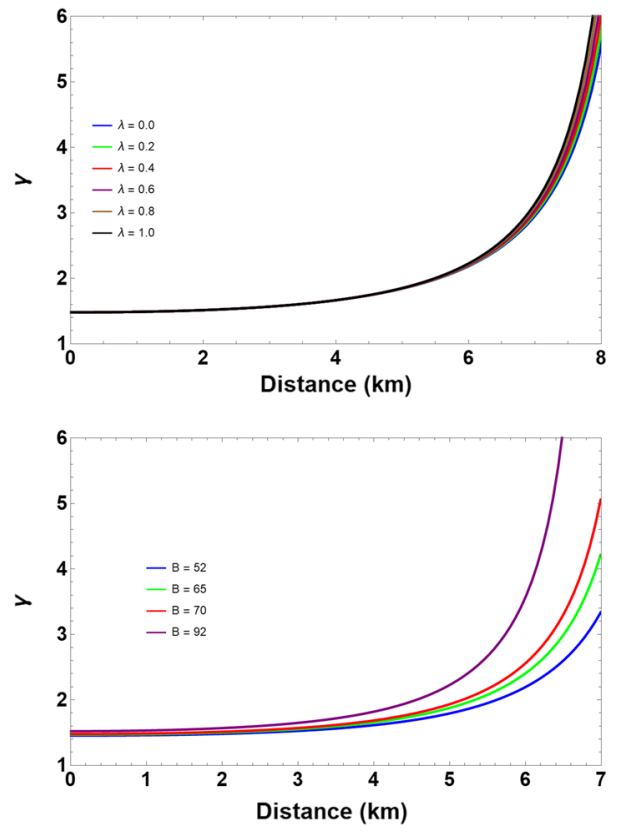

Fig. 4 The plot for adiabatic index, $\gamma$, for an interacting quark EoS with the same set of parameters in Figs. 1 and 2, respectively

of $\lambda$, the maximum masses of QSs increases but the radii decrease. Thus, the compactness of a star is increasing with these decreasing radii, see Fig. 1. However, the case of bag constant $B$ is completely different, where one can see that the existence of $>2.5 M_{\odot}$ is confirmed for less interacting quarks i.e., the lower value of $B$ (see Table 2). In this scenario, we find that our predicted models have maximum masses above $2.5 M_{\odot}$, i.e., in agreement with the observational constraints obtained from GW 190814 data and PSR J0751+1807. In this scenario, we find that our predicted models have maximum masses above $2.5 M_{\odot}$, i.e., in agreement with the observational constraints obtained from GW 190814 data and PSR J0751+1807. Interestingly, our results support the General Relativity claim that NSs cannot have gravitational masses larger than $3 M_{\odot}$. This argument is consistent with the results obtained in [42], where authors have shown that maximum NS masses are likely to be in the lower limits of the range of $2.5 M_{\odot}-3 M_{\odot}$ in the context of $R^{2}$ gravity.

Then, we move on the stability related issues on QSs through static stability criterion and the adiabatic index $\gamma$ which indicate the onset of instability. In particular, we infer the condition $\frac{\partial M\left(\rho_{c}\right)}{\partial \rho_{c}} \lessgtr 0$ by graphical representation (see Fig. 2) where we identify the turning point from stability to instability.

Finally, we move on the stability related issues of QSs through static stability criterion and the adiabatic index $\gamma$, which indicate the onset of instability. In particular, we infer the condition $\frac{\partial M\left(\rho_{c}\right)}{\partial \rho_{c}} \lessgtr 0$ by graphical representation (see Fig. 4) where we identify the turning point from stability to instability. Finally, addressing the critical values of the adiabatic index, $\gamma_{c r}$, our results show that the obtained value of $\gamma>\gamma_{c r}$, which means a stable QS may exist in modified $f(R, T)$ gravity theory.

Acknowledgements A. Pradhan thanks IUCCA, Pune, India for providing facilities under the associateship programmes. T. Tangphati was supported by King Mongkut's University of Technology Thonburi's Post-doctoral Fellowship.

Data Availability Statement This manuscript has no associated data or the data will not be deposited. [Authors' comment: This is a theoretical study and the results can be verified from the information available.]

Open Access This article is licensed under a Creative Commons Attribution 4.0 International License, which permits use, sharing, adaptation, distribution and reproduction in any medium or format, as long as you give appropriate credit to the original author(s) and the source, provide a link to the Creative Commons licence, and indicate if changes were made. The images or other third party material in this article are included in the article's Creative Commons licence, unless indicated otherwise in a credit line to the material. If material is not included in the article's Creative Commons licence and your intended use is not permitted by statutory regulation or exceeds the permitted use, you will need to obtain permission directly from the copyright holder. To view a copy of this licence, visit http://creativecomm ons.org/licenses/by/4.0/.

Funded by $\mathrm{SCOAP}^{3}$.

\section{References}

1. B.P. Abbott et al., LIGO Scientific and Virgo, Phys. Rev. Lett. 116, 061102 (2016)

2. K. Akiyama et al., Event Horizon Telescope, Astrophys. J. Lett. 875, L5 (2019)

3. A.A. Starobinsky, Phys. Lett. B 91, 99 (1980)

4. K.V. Staykov, D.D. Doneva, S.S. Yazadjiev, K.D. Kokkotas, Phys. Rev. D 92, 043009 (2015)

5. K.V. Staykov, D.D. Doneva, S.S. Yazadjiev, K.D. Kokkotas, JCAP 10, 006 (2014)

6. T. Harko, F.S.N. Lobo, S. Nojiri, S.D. Odintsov, Phys. Rev. D 84, $024020(2011)$

7. A. Das, F. Rahaman, B.K. Guha, S. Ray, Eur. Phys. J. C 76, 654 (2016)

8. S. Hansraj, Eur. Phys. J. C 78, 700 (2018)

9. J.M.Z. Pretel, S.E. Jorás, R.R.R. Reis, J. Arbañil, JCAP 08, 055 (2021)

10. P. Rej, P. Bhar, M. Govender, Eur. Phys. J. C 81, 316 (2021)

11. B.P. Abbott et al., LIGO Scientific and Virgo, Phys. Rev. X 9, 031040 (2019)

12. R. Nair, S. Perkins, H.O. Silva, N. Yunes, Phys. Rev. Lett. 123, 191101 (2019)

13. S.D. Odintsov, V.K. Oikonomou, F.P. Fronimos, Nucl. Phys. B 958, $115135(2020)$

14. A.V. Astashenok, S. Capozziello, S.D. Odintsov, V.K. Oikonomou, Phys. Lett. B 811, 135910 (2020)

15. M.X. Xu, T. Harko, S.D. Liang, Eur. Phys. J. C 76, 449 (2016)

16. H. Shabani, A.H. Ziaie, Eur. Phys. J. C 77, 507 (2017)

17. M.J.S. Houndjo, O.F. Piattella, Int. J. Mod. Phys. D 21, 1250024 (2012)

18. S. Capozziello, F.S.N. Lobo, J.P. Mimoso, Phys. Rev. D 91, 124019 (2015)

19. P.H.R.S. Moraes, J.D.V. Arbañil, M. Malheiro, JCAP 06, 005 (2016) 
20. B.C. Barrois, Nucl. Phys. B 129, 390 (1977)

21. M.G. Alford, K. Rajagopal, F. Wilczek, Phys. Lett. B 422, 247 (1998)

22. D. Bailin, A. Love, Nucl. Phys. B 190, 175 (1981)

23. T. Schäfer, F. Wilczek, Phys. Rev. D 60, 114033 (1999)

24. M.G. Alford, J. Berges, K. Rajagopal, Nucl. Phys. B 558, 219 (1999)

25. M.G. Alford, K. Rajagopal, F. Wilczek, Nucl. Phys. B 537, 443 (1999)

26. A. Schmitt, Lect. Notes Phys. 811, 1 (2010)

27. M.G. Alford, A. Schmitt, K. Rajagopal, T. Schäfer, Rev. Mod. Phys. 80, 1455 (2008)

28. R. Rapp, T. Schäfer, E.V. Shuryak, M. Velkovsky, Ann. Phys. 280, $35(2000)$

29. M.G. Alford, K. Rajagopal, S. Reddy, F. Wilczek, Phys. Rev. D 64, 074017 (2001)

30. M. Alford, S. Reddy, Phys. Rev. D 67, 074024 (2003)

31. A. Steiner, S. Reddy, M. Prakash, Phys. Rev. D 66, 094007 (2002)
32. G. Lugones, J.E. Horvath, Phys. Rev. D 66, 074017 (2002)

33. C.V. Flores, G. Lugones, Phys. Rev. C 95, 025808 (2017)

34. E. Farhi, R.L. Jaffe, Phys. Rev. D 30, 2379 (1984)

35. R. Abbott et al., LIGO Scientific and Virgo, Astrophys. J. Lett. 896, L44 (2020)

36. D.J. Nice et al., Astrophys. J. 634, 1242 (2005)

37. B.K. Harrison, Gravitational Theory and Gravitational Collapse (University of Chicago Press, Chicago, 1965)

38. Y.B. Zeldovich, I.D. Novikov, Relativistic Astrophysics, Vol. I: Stars and Relativity (University of Chicago Press, Chicago, 1971)

39. S. Chandrasekhar, Phys. Rev. Lett. 12, 114 (1964)

40. S. Chandrasekhar, Astrophys. J. 140, 417 (1964)

41. E.N. Glass, A. Harpaz, Mon. Not. R. Astron. Soc. 202, 1 (1983)

42. A.V. Astashenok, S. Capozziello, S.D. Odintsov, V.K. Oikonomou, arXiv:2111.14179 [gr-qc] 